\title{
Imaging and Clinical Data of Placental Site Trophoblastic Tumor: A Case Report
}

\author{
Maryam Niknejadi, ${ }^{1}$ Firoozeh Ahmadi, ${ }^{1,}$ and Farnaz Akhbari ${ }^{1}$ \\ ${ }^{1}$ Department of Reproductive Imaging , Reproductive Biomedicine Research Center, Royan Institute for Reproductive Biomedicine, ACECR, Tehran, Iran \\ "Corresponding author: Firoozeh Ahmadi, Department of Reproductive Imaging, Reproductive Biomedicine Research Center, Royan Institute for Reproductive Biomedicine, \\ ACECR, Tehran, Iran. E-mail: dr.ahmadi1390@gmail.com
}

Received 2014 February 23; Revised 2014 October 01; Accepted 2014 October 25.

\begin{abstract}
Placental site trophoblastic tumor (PSTT) is a very rare variant of gestational trophoblastic tumor. It can occur after normal termination of pregnancy or spontaneous abortion and ectopic or molar pregnancy. There is a wide range of clinical manifestations from a benign condition to an aggressive disease with fatal outcome. One of the most important characteristics of PSTT, unlike other forms of gestational trophoblastic diseases (GTD) is the presence of low beta-subunit of human chorionic gonadotropin ( $\beta$-hCG) levels because it is a neoplastic proliferation of intermediate trophoblastic cells. However, human placental lactogen (hPL) is increased on histologic section and in the serum of patients too. We present a case of PSTT and discuss the differential diagnosis in order to further familiarize physicians with the diagnosis and treatment of this disease. It has a varied clinical spectrum and usually presents with irregular vaginal bleeding or amenorrhea. Diagnosis is confirmed by dilatation and curettage (D and C) and hysterectomy. Because chemotherapy is not effective, surgery is the cornerstone of treatment. This case is presented because it is a rare neoplasm with different treatments and it should be differentiated from molar pregnancy.
\end{abstract}

Keywords: Placental Site Trophoblastic Tumor, Ultrasound

\section{Introduction}

Placental site trophoblastic tumor (PSTT) is a slow growing malignant intermediate trophoblastic tumor and a rare form of gestational trophoblastic disease (GTD) (1). PSTT is the least common form of gestational trophoblastic neoplasia composed of predominantly intermediate trophoblasts (Figure 1). Since 1981, about 150 cases have been reported in literature. The incidence of PSTT is about 1/100000 pregnancies and it constitutes about 1 - $2 \%$ of all GTDs (1). For the first time, Larsen et al. proposed the term of PSTT in 1991. It was considered as an exaggerated form of reaction of the placental bed (2). PSTT can occur several months, or even years, after the pregnancy including molar and ectopic, miscarriage and abortion, or a full term normal pregnancy (3).

Unlike other forms of GTD, serum beta-subunit of human chorionic gonadotropin ( $\beta$-hCG) is low, because it is originated from a neoplastic proliferation of intermediate trophoblastic cells.

It may secrete human placental lactogen (hPL) and result in a false-positive pregnancy test (4). The most common presenting symptoms are irregular vaginal bleeding with or without preceding amenorrhea. Diagnosis will be confirmed by D and C hysterectomy. Assessment of metastasis plays a significant role in treatment choices and ther- apeutic procedures.

In this study, the karyotype of PSTT is diploid but in some studies the case of triploidy and tetraploidy have been reported (5). Sex chromosome analysis of patients suggests a unique genetic basis for the development of PSTT that involves the paternal X chromosome (6). We present a case of PSTT and will discuss the differential diagnosis in order to further familiarize physicians with the diagnosis and treatment of this disease. Due to its rarity and varied biological behavior, it presents a specific diagnosis as it is distinct from other GTNs.

\section{Case Presentation}

A 22-year-old G1P1 woman presented to Royan institute with a recent history of several months of amenorrhea. The last delivery was 3 years ago. Physical abdominal examination was normal. Pelvic examination showed a palpable uterine mass. Hysterosalpingography (HSG) revealed a uterine cavity with irregular contours. We used HSG in the process of assisted reproductive technique (ART) cycle.

Then after she underwent transvaginal ultrasonography (TVUS), a depicted heterogenic and irregular illdefined mass lesion $(37 \times 29 \mathrm{~mm})$ invading the anterior wall of the uterus body was detected. Uterine dimensions were $73 \times 43 \times 57 \mathrm{~mm}$ with a global shape. In the 
uterus, a tumorous mass was found that extended to about half of the myometrium. A thin endometrial line was seen indistinctly; therefore, differentiation between myometrium and endometrium was difficult (Figure 2). On color Doppler examination, the mass was hypervascular (Figure 3). Magnetic resonance imaging (MRI) revealed an indistinct moderate size, heterogonous mass lesion in the anterior wall of the uterus with a thin endometrium (Figure 4).

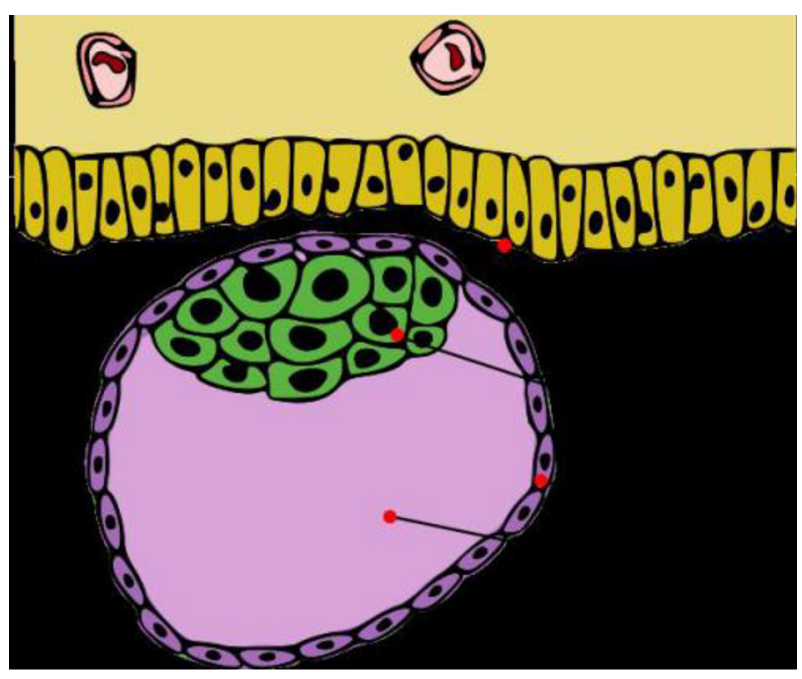

Figure 1. Schematic features of the blastocyst and trophoblast. The blastocyst comprises an inner cell mass that subsequently forms the embryo and the outer layer called trophoblast that provides nutrients to the embryo and develops into a large part of the placenta.

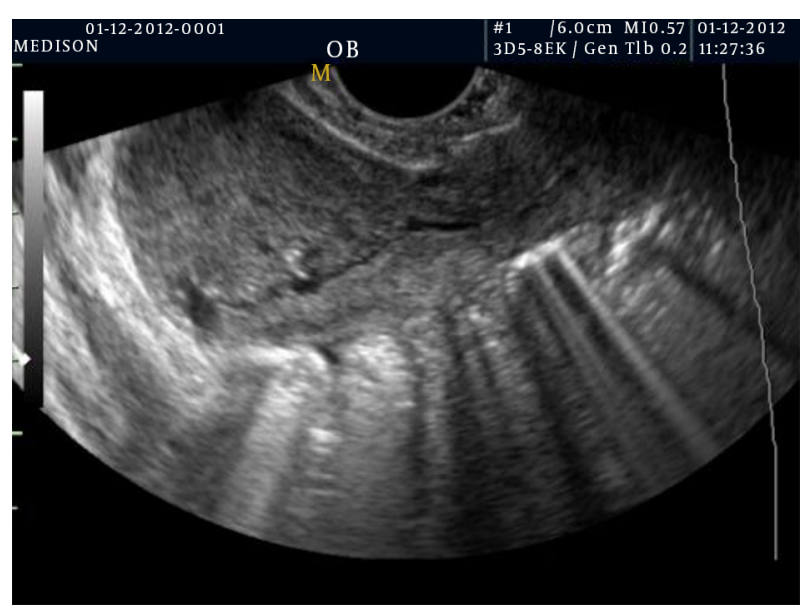

Figure 2. A 22-year-old G1P1 woman with a recent history of amenorrhea and palpable pelvic mass. Transvaginal ultrasonography shows a heterogenic and irregular ill-defined mass lesion $(37 \times 29 \mathrm{~mm})$ invading the anterior wall of the uterus body.

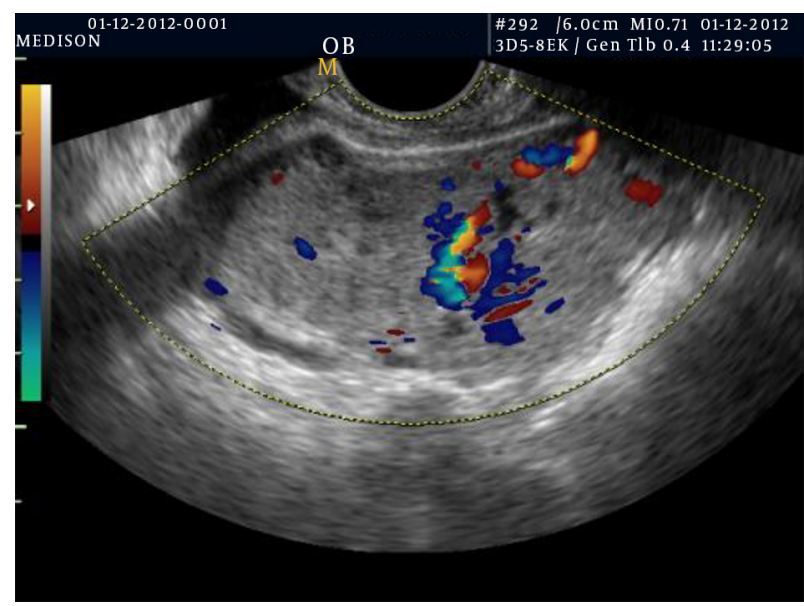

Figure 3. Color Doppler sonography depicts hypervascularity in the area of the tumor.

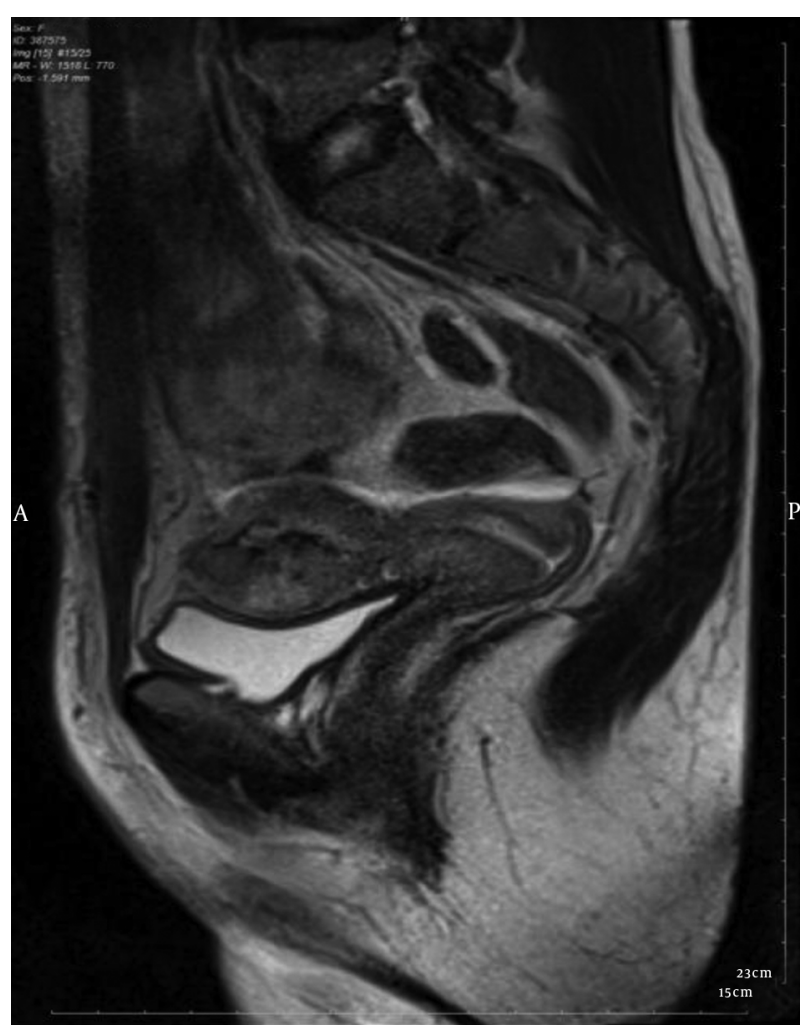

Figure 4. Sagittal T2 weighted image of the pelvis shows indistinct heterogeneous abnormal signal changes in the anterior wall with a thin endometrium.

Based on these findings, invasive mole or choriocarcinoma was suspected, but $\beta$-hCG was low (50 IU/mL). Therefore, the probability of PSTT was proposed. In order to determine the final diagnosis, therapeutic curettage was 
done in Royan institute. The result of the histopathological specimen showed focal areas of atypical endometrium. On the basis of clinical, laboratory, ultrasound findings and finally the pathology result, the case was diagnosed as PSTT. Laparotomy with total abdominal hysterectomy was performed. The postoperative recovery period was normal and she did not have postoperative chemotherapy.

\section{Discussion}

PSTT is a very rare and unique form of GTD (7). It is defined as a slow growing malignancy, malignant tumors of intermediate trophoblasts that form a nodule in the endometrium and myometrium (8). The cells of intermediate trophoblasts constitute a transitional form between cytotrophoblasts and syncytiotrophoblasts, their physiological role is to form the placental bed. They may also have a pathological role in three diseases, one of which is called PSTT.

In 1976, Kurman et al. described 12 patients with this distinct form of trophoblastic disease (9). Guvendag Guven et al. called this disease placental site trophoblastic tumor to explain the malignant potential of this tumor. Since then, about 150 cases have been reported in the literature (10).

The etiology, epidemiology and risk factor for PSTT are unknown (1). Cole et al. reported that hCG and its free betasubunit could be used as a reliable marker for the diagnosis of PSTT (11). However, a study conducted by Bower et al. in 1996 reported that tumor cells are positive for hPL and serum hCG is a less sensitive tumor marker in PSTT $(6,12)$. Hence, a raised hPL level and a normal to slightly elevated hCG level are valuable clues in the diagnosis of PSTT.

Symptoms may appear weeks or years after the previous pregnancy. The first symptoms of the disease are usually seen after delivery or abortion. Vaginal bleeding or amenorrhea is the usual mode of presentation, as it appeared in the present case too.

The world health organization (WHO) classified four classes of GTD. The first class is the hydatidiform mole, the second is the invasive mole, and the third and fourth are choriocarcinoma and PSTT, respectively. PSTT is divided into two types, hypervascular and relatively hypovascular types.

The important role of imaging particularly color Doppler sonography in the diagnosis of PSTT is to clarify the vascularity of the tumor (13). The hypervascularity was confirmed by color Doppler. D and C should be avoided because after dilatation and curettage, severe bleeding has been reported. On the other hand, in the localized hypovascular type, conservative surgery may be possible.
According to sonographic and HSG findings, this disease must be differentiated from: (i) invasive mole, (ii) choriocarcinoma, (iii) organized retained previous placenta from previous delivery, (iv) uterus tumor lesion such as epithelioid type of leiomyosarcoma, (v) endometrial polyp, and (vii) placental site nodule (14). In choriocarcinoma, $\beta$-hCG titer is high, but in PSTT, it is low. Therefore, serum $\beta$-hCG titer could be used as a clue for differentiation between PSTT and choriocarcinoma.

Generally, ultrasonography illustrates an echogenic mass that can involve the endometrium and myometrium. Doppler scan can show that there are more blood vessels than usual in the area of the tumor (similar to the presented case).

The prognostic factors are age, interval from antecedent pregnancy ( $>4$ years indicating poor prognosis), FIGO staging and mitotic count $(>5 / 10$ HPF high power field) and metastasis $(13,15)$. In the presented case, a diagnosis of PSTT was made 3 years after her previous delivery. Poor prognosis was reported in cases with distant metastasis or delayed treatment. On the other hand, in cases which are localized to the uterus, and in which the interval between the previous pregnancy and treatment is less than 2 years, good prognosis is expected. The majority of cases of PSTT exhibit benign manifestations and are confined to the uterus at the time of the diagnosis, but $10-15 \%$ of them are clinically malignant and have metastasizes and are resistant to chemotherapy $(8,10)$. Recurrence of PSTT metastases has been reported in 10\% (10).

When PSTT is diagnosed on endometrial curettage, a thorough metastatic work-up should be undertaken. Due to its poor response to chemotherapy, a diagnosis of nonmetastatic PSTT should be followed by prompt hysterectomy.

Advances in chemotherapeutic regimens have improved clinical response in metastatic disease. The firstline treatment option is surgery, but for patients desiring future childbearing, D and C and chemotherapy is an alternative choice.

In conclusion, PSTT is a well-recognized but rare form of GTD with variable presentations. Most of the tumors are localized in the uterus. Due to its rarity and variable manifestations, it presents a diagnostic challenge and treatment dilemma. It is important to make this specific diagnosis, as it is distinct from other GTNs. The aim of presenting this case of uterine PSTT was to illustrate the difficulties in the diagnosis of this tumor and how this led to delay in the appropriate management. Hysterectomy seems to be the appropriate treatment of a patient with confined tumor in the uterus, but metastatic patients also need chemotherapy. 


\section{Acknowledgments}

The authors wish to thank the imaging department staff for their assistance in figure preparation.

\section{References}

1. Ajithkumar TV, Abraham EK, Rejnishkumar R, Minimole AL. Placental site trophoblastic tumor. Obstet Gynecol Surv. 2003;58(7):484-8. doi: 10.1097/01.OGX.0000077466.40895.32. [PubMed: 12832940].

2. Larsen LG, Theilade K, Skibsted L, Jacobsen GK. Malignant placental site trophoblastic tumor. A case report and a review of the literature. APMIS Suppl. 1991;23:138-45. [PubMed: 1652995].

3. Fisher RA, Soteriou BA, Meredith L, Paradinas FJ, Newlands ES. Previous hydatidiform mole identified as the causative pregnancy of choriocarcinoma following birth of normal twins. Int J Gynecol Cancer. 1995;5(1):64-70. [PubMed: 11578456].

4. Whitney KA. Placental site trophoblastic tumor. Am J Nurs. 2009;109(12):32-7. doi: 10.1097/01.NAJ.0000365176.62954.11. [PubMed: 19935163] quiz 38.

5. Xue WC, Guan XY, Ngan HY, Shen DH, Khoo US, Cheung AN. Malignant placental site trophoblastic tumor: A cytogenetic study using comparative genomic hybridization and chromosome in situ hybridization. Cancer. 2002;94(8):2288-94. doi: 10.1002/cncr.10424. [PubMed: 12001129].

6. Hui P, Parkash V, Perkins AS, Carcangiu ML. Pathogenesis of placental site trophoblastic tumor may require the presence of a paternally derived X chromosome. Lab Invest. 2000;80(6):965-72. [PubMed: 10879746].
7. Kim SJ. Placental site trophoblastic tumour. Best Pract Res Clin Obstet Gynaecol. 2003;17(6):969-84. [PubMed: 14614893].

8. Behtash N, Karimi Zarchi M. Placental site trophoblastic tumor.JCancer Res Clin Oncol. 2008;134(1):1-6. doi: 10.1007/s00432-007-0208-y. [PubMed: 17701427].

9. Kurman RJ, Scully RE, Norris HJ. Trophoblastic pseudotumor of the uterus: an exaggerated form of "syncytial endometritis" simulating a malignant tumor. Cancer. 1976;38(3):1214-26. [PubMed: 182351].

10. Guvendag Guven ES, Guven S, Esinler I, Ayhan A, Kucukali T, Usubutun A. Placental site trophoblastic tumor in a patient with brain and lung metastases. Int J Gynecol Cancer. 2004;14(3):558-63. doi: 10.1111/j.1048891x.2004.014322.x. [PubMed: 15228435].

11. Cole LA, Khanlian SA, Muller CY, Giddings A, Kohorn E, Berkowitz R. Gestational trophoblastic diseases: 3. Human chorionic gonadotropin-free beta-subunit, a reliable marker of placental site trophoblastic tumors. Gynecol Oncol. 2006;102(2):160-4. doi 10.1016/j.ygyno.2005.12.046. [PubMed: 16631918].

12. Bower M, Paradinas FJ, Fisher RA, Nicholson SK, Rustin GJ, Begent RH, et al. Placental site trophoblastic tumor: molecular analysis and clinical experience. Clin Cancer Res. 1996;2(5):897-902. [PubMed: 9816247].

13. Sumi Y, Ozaki Y, Shindoh N, Katayama H. Placental site trophoblastic tumor: imaging findings. Radiat Med. 1999;17(6):427-30. [PubMed: 10646979].

14. Shih IM, Kurman RJ. Ki-67 labeling index in the differential diagnosis of exaggerated placental site, placental site trophoblastic tumor, and choriocarcinoma: a double immunohistochemical staining technique using Ki-67 and Mel-CAM antibodies. Hum Pathol. 1998;29(1):2733. [PubMed: 9445130].

15. Newlands ES, Bower M, Fisher RA, Paradinas FJ. Management of placental site trophoblastic tumors. J Reprod Med. 1998;43(1):53-9. [PubMed: 9475150]. 